\title{
The Effect of Processing Additives on Energetic Disorder in Highly Efficient Organic Photovoltaics: A Case Study on PBDTTT-C- T:PC71BM
}

Feng Gao, Scott Himmelberger, Mattias Andersson, David Hanifi, Yuxin Xia, Shaoqing Zhang, Jianpu Wang, Jianhui Hou, Alberto Salleo and Olle Inganäs

\section{Linköping University Post Print}

\section{Tweet}

N.B.: When citing this work, cite the original article.

Original Publication:

Feng Gao, Scott Himmelberger, Mattias Andersson, David Hanifi, Yuxin Xia, Shaoqing Zhang, Jianpu Wang, Jianhui Hou, Alberto Salleo and Olle Inganäs, The Effect of Processing Additives on Energetic Disorder in Highly Efficient Organic Photovoltaics: A Case Study on PBDTTTC-T:PC71BM, 2015, Advanced Materials, (27), 26, 3868-3873.

http://dx.doi.org/10.1002/adma.201405913

Copyright: Wiley-VCH Verlag

http://www.wiley-vch.de/publish/en/

Postprint available at: Linköping University Electronic Press http://urn.kb.se/resolve?urn=urn:nbn:se:liu:diva-120340 


\section{WILEY-VCH}

DOI: $10.1002 /(($ please add manuscript number $))$

Article type: Communication

The effect of processing additives on energetic disorder in highly efficient organic photovoltaics: A case study on PBDTTT-C-T:PC 71 BM

Feng Gao, ${ }^{1 *}$ Scott Himmelberger, ${ }^{2}$ Mattias Andersson, ${ }^{1}$ David Hanifi, ${ }^{2}$ Yuxin Xia,,${ }^{1}$ Shaoqing Zhang, ${ }^{3}$ Jianpu Wang, ${ }^{*}$ Jianhui Hou,,$^{3 *}$ Alberto Salleo, ${ }^{2 *}$ and Olle Inganäs ${ }^{1}$

Dr. Feng Gao, Dr. Mattias Andersson, Mr. Yuxin Xia, and Prof. Olle Inganäs

Biomolecular and Organic Electronics, IFM, Linköping University, Linköping 58183,

Sweden

E-mail: fenga@ifm.liu.se

Mr. Scott Himmelberger, Mr. David Hanifi, and Prof. Alberto Salleo

Materials Science and Engineering, Stanford University, Stanford, CA 94305, USA

E-mail: asalleo@stanford.edu

Ms. Shaoqing Zhang and Prof. Jianhui Hou

State Key Laboratory of Polymer, Physics and Chemistry, Institute of Chemistry, Chinese Academy of Sciences, Beijing 100190, P. R. China

E-mail: hjhzlz@iccas.ac.cn

Prof. Jianpu Wang

Key Laboratory of Flexible Electronics (KLOFE) \& Institute of Advanced Materials (IAM), National Synergistic Innovation Centre for Advanced Materials (SICAM), Nanjing Tech University, 30 South Puzhu Road, Nanjing 211816, China

E-mail: iamjpwang@njtech.edu.cn

Keywords: organic photovoltaics, additives, energetic disorder, morphology

The power conversion efficiency (PCE) of organic photovoltaics (OPVs) has approached

$10 \%$ in highly efficient devices. ${ }^{[1-3]}$ A milestone in the development of OPVs has been the introduction of processing additives which may significantly increase the device performance in many photovoltaic blends. These additives include high boiling point solvents, ${ }^{[4]}$ selective solvents (e.g. alkane dithiol), ${ }^{[1]}$ polar non-solvents, ${ }^{[5]}$ and very frequently diiodooctane (DIO). ${ }^{[6]}$ In spite of widespread application of additives in OPVs, a mechanistic understanding of the additive-induced efficiency enhancement remains limited. ${ }^{[7]}$ 


\section{WILEY-VCH}

One of the major ways by which additives improve OPV device performance is by helping to optimize the active layer morphology. Since OPVs are made from a blend of nanostructured donor (e.g. polymer) and acceptor (e.g. fullerene) components, the morphology at the nanoscale is of critical importance for the device performance. While additives have clearly been shown to have dramatic impacts on OPV nanostructure, the specific effects can vary widely depending on the particular donor/acceptor system used. In some systems, additives have been shown to increase the domain size of donor and acceptor components, enhancing charge transport and decreasing bimolecular recombination of separated charge carriers. ${ }^{[6,8]}$ In others, additives play an important role in dissolving the fullerene aggregates and/or improving the crystallinity of the polymer phase, enhancing charge separation. ${ }^{[9-11]}$ Very recently, Guo et al. showed that additives help to optimize surface compositions, enabling a polymer-rich composition close to the anode which enhances charge collection. ${ }^{[12]}$ These previous reports demonstrate that additives play a critical role in optimizing OPV morphology, which affects all the steps involved in photon-toelectron conversion, including charge separation, charge recombination, charge transport, and charge collection. ${ }^{[13,14]}$

In addition to morphology and geometrical disorder, another parameter that is vitally important to OPV performance is the energetic disorder. It has been demonstrated that higher levels of energetic disorder increase recombination and are detrimental to device performance. ${ }^{[15]}$ Recent spectroscopic measurements and quantum-mechanical calculations also indicate that lower levels of energetic disorder in the fullerene phase can suppress recombination of triplet charge-transfer states, and hence enhance device performance. ${ }^{[16,17]}$ However, little is known concerning the effect of additives on the energetic disorder in OPVs.

In this communication, we investigate how additives affect the energetic disorder in a polymer:fullerene model system consisting of a benzodithiophene-based copolymer 


\section{WILEY-VCH}

((poly(4,8-bis(5-(2-ethylhexyl)-thiophene-2-yl)-benzo[1,2-b:4,5-b']dithiophene-alt-

alkylcarbonyl-thieno[3,4-b]thiophene)), PBDTTT-C-T) and phenyl-C 71 -butyric-acid-methyl

ester ( $\left.\mathrm{PC}_{71} \mathrm{BM}\right)$ (see Figure $\mathrm{S} 1$ for $J$ - $V$ curves). Benzodithiophene-based copolymers have found widespread applications in highly efficient OPV devices. ${ }^{[18]}$ Based on temperaturedependent mobility measurements, we demonstrate that the additive DIO lowers energetic disorder in the blend. We show that the improvement in energetic order occurs primarily for electron transport in acceptor domains, while the disorder for holes is relatively unaffected by DIO. The ability of DIO to lower the energetic order is confirmed by highly sensitive measurements of the weak charge-transfer state emission. Wide-angle (WAXS) and smallangle X-ray scattering (SAXS) measurements suggest the origin of this reduced energetic disorder is due to increased aggregation and a larger average fullerene domain size together with purer phases upon addition of DIO.

The most straightforward approach to investigate the energetic disorder in organic semiconductors is to make temperature dependent mobility measurements. In organic semiconductors, charge carrier transport occurs through a distribution of states via a hopping process, and the mobility is not a constant due to the energetic disorder. Instead, it depends on both electric field and carrier concentrations. ${ }^{[19]}$ As the electric field increases, a greater number of states are accessible to charge carriers, resulting in an increase in the mobility. In addition, with increasing carrier concentration the average energy of the carriers increase, making the carrier hopping process easier and giving rise to a carrier-density-dependent mobility. ${ }^{[19]}$ The dependence of mobility on carrier density helps to explain important observations in organic semiconductors. For example, the mobility enhancement commonly observed in a transistor architecture relative to measurements done in a diode structure for the same material is due to a significantly higher carrier concentration for the former. ${ }^{[20]}$ 


\section{WILEY-VCH}

In order to extract the energetic disorder value from electrical measurements, a numerical model for the mobility is required. Pasveer et al. developed a model, known as the extended Gaussian disorder model (EGDM), to account for the effects of the carrier concentration on the mobility in space-charge-limited (SCL) $J$-V curves. ${ }^{[21]}$ Greenham and co-workers demonstrated that the EGDM could be applied in single-carrier devices as well as blends. ${ }^{[22,23]}$ In these studies, it was found that the field enhancement factor predicted by the Pasveer model failed to reproduce the single-carrier current densities, and that a Poole-Frenkel-type field enhancement of the mobility was used instead. The discrepancy was suggested to arise from the presence of correlated disorder. ${ }^{[22]}$ Following Greenham's approach to parametrize the mobility, $J-V$ curves can be numerically solved based on the drift-current equations and the Poisson's equation (see the SI for details). ${ }^{[24,25]}$ In this way, the energetic disorder can be quantified by measuring and modelling $J$ - $V$ curves of single-carrier devices over a range of temperatures. Though not the focus of this paper, we note that in addition to the EGDM, there are other models describing charge transport in organic semiconductors, e.g. band-like transport with multiple trapping. ${ }^{[26-28]}$

$J$ - $V$ curves of electron-only devices, processed with and without DIO, taken at different temperatures are shown in Figure 1 (a) and (b) (open symbols). For each temperature, the $J$ - $V$ curves are fitted using the EGDM with a Poole-Frenkel-type field enhancement (see the SI for details). The fits (solid lines in Figure 1a and 1b) are achieved with a Gaussian disorder width of $\sigma=75 \mathrm{meV}$ for the device with DIO and $90 \mathrm{meV}$ for that without DIO. It is clear that the addition of DIO decreases the energetic disorder of electrons. We note that the situation might be different for different blends. For example, in polythieno[3,4-b]-thiophene-cobenzodithiophene (PTB7): $\mathrm{PC}_{71} \mathrm{BM}$ blends, it was found that the addition of DIO leads to a reduced electron mobility, presumably due to reduced size of the fullerene-rich domains in that case ${ }^{\left[{ }^{[2]}\right.}$ For hole transport, the addition of DIO has a negligible effect on the hole current 


\section{WILEY-VCH}

for all temperatures (Figure S2). This observation is consistent with a previous report on PTB7, where no effect on the hole transport was observed upon addition of DIO. ${ }^{[29]}$ By fitting the curves at different temperatures, we obtain a Gaussian disorder width $\sigma$ of $70 \mathrm{meV}$ for holes (Figure 1c) in devices with and without DIO. We also plot the relation between the field activation parameter $\gamma$ and temperature $T$ for all the devices in Figure S3. Simulations based on spatially correlated disorder predict a temperature dependence of $\gamma \propto T^{\alpha \alpha}$, ${ }^{[30]}$ with $\alpha$ varying between 1 and 2 . The value of $\alpha$ depends on the degree of correlation, with completely uncorrelated disorder giving an $\alpha$ value of 2. The fitting in Figure S3 shows $\alpha=$ 1.5 (solid lines), suggesting that the disorder in this system may be correlated.

In order to confirm the disorder values obtained from the single-carrier diode configurations, we performed a complementary experiment in a transistor configuration. The transistor data can be evaluated in a more straightforward manner, ${ }^{[31]}$ although charge transport occurs in a different direction compared with that in OPVs. The transistor measurements are fully consistent with the diode data. They show a nearly identical hole mobility for devices with and without DIO for all the measured temperatures, while there is a marked decrease of the temperature dependence of the electron mobility in samples with DIO, indicating a reduced disorder. By evaluating the data using the Gaussian Disorder Model (GDM) and a carrier concentration dependent mobility according to a numerical approximation suggested by Coehoorn et al., ${ }^{[32]}$ qualitatively similar disorder numbers to the diode measurements are obtained. The $\sigma$ values are higher in the case of the transistor structure: $79 \mathrm{meV}$ for holes (both with and without DIO), $123 \mathrm{meV}$ for electrons without DIO and $91 \mathrm{meV}$ for electrons with DIO. Higher $\sigma$ values in the transistor structure could be due to the influence of water at the dielectric interface of the transistor ${ }^{[31]}$ or due to the fact that the interface is more disordered. ${ }^{[33]}$ We note that other polymer:fullerene blends react in different ways to additives. For example, it was found that 1,8-octanedithiol can increase the hole 


\section{WILEY-VCH}

mobility of a polymer:fullerene blend in the transistor configuration, where a benzothiadiazole-containing copolymer is employed. ${ }^{[34]}$

Further information concerning the energetic disorder is obtained by measuring the weak emission from charge-transfer (CT) states using highly sensitive techniques. CT emission happens at the interfaces between the donor and acceptor, providing rich information on the energetic ordering of these two phases. ${ }^{[35,36]}$ The electroluminescence (EL) of the devices with and without DIO are shown in Figures 2 (a) and 2 (b), respectively. Since the EL of both pure PBDTTT-C-T and pure $\mathrm{PC}_{71} \mathrm{BM}$ is above $1.5 \mathrm{eV},{ }^{[37]}$ we attribute the $\mathrm{EL}$ in the blend to the emission from CT states. ${ }^{[38]}$ The CT EL spectra show a slight blue shift with increasing bias in both devices, as demonstrated in other blends as well. ${ }^{[38,39]}$ It is possible to explain such a shift with state-filling effects, which would require electron densities (the most disordered phase) above $10^{-5}$ carriers per site for the measured levels of disorder ${ }^{[36]}$ Another possible explanation for the EL shift could be energetic relaxation, where the average carrier energy decreases with time. This time is usually comparable to the transit time for the given conditions. ${ }^{[40]}$ It is obvious that the EL of the device without DIO shows a stronger dependence on the injected current, which is consistent with a higher disorder irrespective of if it is due to state-filling effects or energetic relaxation. Due to the spectrum shift caused by energetic disorder in CT EL measurements, we consider the CT absorption measurement to be a more suitable estimation of the CT state energy (Figure S4).

In addition to CT EL, we also show photoluminescence (PL) of CT states in the same figure. The PL of the blend is usually dominated by the emission from pure components, and the emission from CT states only appears as a shoulder (Figure S5). The CT emission can be quenched by applying a negative bias, enabling us to obtain solely the CT emission by subtracting the emission of the blend at a negative bias ( $-2 \mathrm{~V}$ in our case) from that at the open-circuit voltage. CT PL shows a blue shift compared with CT EL, consistent with 


\section{WILEY-VCH}

previous reports. ${ }^{[35]}$ This behaviour can be explained by taking the energetic disorder into consideration. In EL measurements, electrons and holes have relaxed towards their equilibrium energies before meeting and recombining, while in PL measurements the CT emission occurs at, or close to, the point of excitation before most of the relaxation has occurred ${ }^{[35]}$ In the blend without DIO, the shift between EL and PL is larger than that with DIO, again indicating that energetic disorder is larger in the blend without DIO, and agreeing well with our other findings.

In order to understand the origin of the effect of DIO on energetic disorder in OPVs, we performed X-ray diffraction (XRD) measurements on the blend films. WAXS data shows a peak at q 0.3 $\AA^{-1}$, consistent with alkyl stacking in the polymer, as well as peaks at $\mathrm{q} \sim 0.6 \AA^{-1}$ and $\mathrm{q} \sim 1.4 \AA^{-1}$ arising from the fullerene (Figure $3 \mathrm{a}$ and Figure S6). Upon addition of DIO, the intensity of the alkyl stacking peak increases dramatically while the width decreases. An examination of the angular distribution of X-ray intensity reveals that the polymer orientation is nearly isotropic and remains relatively unchanged after addition of DIO, indicating that the increased intensity is representative of greater crystallinity in PBDTTT-C-T. The coherence length in the alkyl stacking direction, as calculated by the Scherrer equation, increases from $5.2 \mathrm{~nm}$ to $10.1 \mathrm{~nm}$ while the coherence length for the fullerene also increases from $2.2 \mathrm{~nm}$ to $2.8 \mathrm{~nm}$. Additionally, a weak peak consistent with pi stacking in PBDTTT-C-T becomes visible near $\mathrm{q} \sim 1.6 \AA^{-1}$ as a shoulder on the right of the fullerene peak at $\mathrm{q} \sim 1.4 \AA^{-1}$. Complementary SAXS data were obtained and are shown for the films cast with and without DIO in Figure $3 b$ and Figure S7. The data shows a shoulder in the Guinier region that shifts to a lower q-value with the addition of DIO. ${ }^{[7]}$ Fitting the SAXS data to a Guinier-Porod empirical model allows us to extract the radius of gyration, $\mathrm{R}_{\mathrm{g}}$, which is a measure related to the effective average size of domains in the film as well as the Porod exponent, m, which describes the shape of these domains. ${ }^{[11,42]}$ We find that the radius of gyration increases from 


\section{WILEY-VCH}

$2.2 \mathrm{~nm}$ to $5.1 \mathrm{~nm}$ upon addition of DIO and the Porod exponent increases from 2.7 to 3.7, indicating that the domains are becoming more three-dimensional. While we cannot distinguish separately the size of polymer and fullerene domains from the SAXS data alone, the WAXS data suggests this increased average domain size is due to enhanced aggregation of both components.

Together, these results indicate that the inclusion of DIO causes increased phase separation and allows the formation of larger domains. As the charge transport and optical measurements show that the energetic disorder is reduced in the fullerene phase for films cast with DIO, we hypothesize that DIO causes increased aggregation for both components (polymer and fullerene) by expelling the fullerene from the polymer phase. This is consistent with the strong increase in the intensity of the alkyl stacking peak and also reconcilable with the still weak pi-stacking peak observed with WAXS upon the addition of DIO. Only very local aggregation is needed for efficient charge transport in many disordered but highly performing semiconducting polymers, considering that the hole transport is predominately along the polymer chains. ${ }^{[43]}$ The improvement in energetic disorder for electron transport coupled with the XRD data suggests that the fullerene domains are growing in size. Small fullerene clusters or even single fullerene molecules in the polymer phase have different energy levels than the main fullerene phase and thus the reduction in these small clusters upon addition of DIO explains the drop in energetic disorder. In addition, the fullerene aggregate quality could improve as well upon the addition of DIO, further decreasing the energetic disorder for electrons. Similar morphological characterization has been performed in other material systems. Resonant soft X-ray scattering (RSoXS), WAXS, and SAXS measurements demonstrated that PTB7:PC ${ }_{71} \mathrm{BM}$ blends adopt a finer length scale upon addition of DIO and indicated the presence of a second, larger domain length scale on the order of $100 \mathrm{~nm} \cdot{ }^{[8,44]}$ As the SAXS measurements used in the present work cannot probe length scales this large, it is 


\section{WILEY-VCH}

possible that a larger domain size is present in our blends; however, the lack of a change in the hole mobility upon addition of DIO suggests dramatic large-scale domain reorganization is unlikely. The effect of processing additives varies widely depending on the system being studied, making it unsurprising that we observe domain growth in the case of PBDTTT-C$\mathrm{T}: \mathrm{PC}_{71} \mathrm{BM} \cdot{ }^{[45]}$

In conclusion, we find that the additive DIO helps to decrease the energetic disorder in the PBDTTT-C-T:PC ${ }_{71} \mathrm{BM}$ blend, where DIO enhances aggregation and phase separation. The reduction in energetic disorder is confirmed independently by temperature-dependent mobility measurements and sensitive measurements of charge-transfer emission. Numerical modelling of mobility measurements in both diode and transistor configurations indicates that the reduction in energetic disorder occurs primarily in the electron-transporting fullerene domains upon the addition of DIO, while that for hole transport is unaffected. WAXS and SAXS measurements suggest the origin of this reduced energetic disorder is due to increased aggregation and a larger average fullerene domain size together with purer phases upon addition of DIO. This work demonstrates a new mechanism for the reasons behind additiveinduced efficiency enhancement and has important implications for future materials and device development, where energetic disorder should be minimized by proper design of the materials and careful optimization of processing conditions.

\section{Experimental Section}

Device fabrication: ITO glass substrates were cleaned using acetone and 2-propanol successively. A $40 \mathrm{~nm}$ thick PEDOT:PSS (Clevious P VP AI 4083, H. C. Stark) layer was spin-coated onto the substrates, which were then annealed at $150{ }^{\circ} \mathrm{C}$ for 30 minutes. The substrates were then transferred into a nitrogen glovebox for the further fabrication steps. A 1:1.5 blend (by weight) of PBDTTT-C-T and $\mathrm{PC}_{71} \mathrm{BM}$ (Nano-C Inc.) was dissolved in 1,2- 


\section{WILEY-VCH}

dichlorobenzene at $80{ }^{\circ} \mathrm{C}$ with a total solution concentration of $25 \mathrm{mg} \mathrm{ml}^{-1}$. This solution was spin-coated at $1000 \mathrm{rpm}$ to yield films with a thickness of $\sim 100 \mathrm{~nm}$. For the blend with DIO, $3 \%$ volume ratio of DIO was added to the solution. $0.8 \mathrm{~nm} \mathrm{LiF}$ and $100 \mathrm{~nm}$ of aluminum were subsequently evaporated onto the active layer under high vacuum (less than $10^{-6}$ mbar), during which a shadow mask was used to define the active area as $\sim 4.5 \mathrm{~mm}^{2}$. To fabricate the single-carrier devices, the procedure was the same, except that different top and bottom contacts were chosen so that only a single carrier species can be injected into the active layer. For the hole-only devices, $\mathrm{LiF} / \mathrm{Al}$ top electrode in the normal structure was replaced by $\mathrm{MoOx}$ $(8 \mathrm{~nm}) / \mathrm{Al}(100 \mathrm{~nm})$, while for the electron-only devices the PEDOT:PSS bottom contact was replaced by a $\mathrm{ZnO}(40 \mathrm{~nm})$ film. Transistors were made by spin-coating the active layers on top of photolithographically pre-patterned field-effect transistor substrates with a $\mathrm{SiO}_{2}$ gate insulator and gold electrodes in the same way as for the diodes.

Temperature dependent measurements: Single-carrier devices were mounted in a liquidnitrogen cryostat for temperature-dependent measurements. The $J-V$ curves was measured using a Keithley 2400 source meter at temperatures ranging from $170 \mathrm{~K}$ to room temperature. The temperature was monitored and controlled using a LakeShore 330 Autotuning Temperature Controller. Transistors were measured in a micromanipulator equipped high vacuum probe station using a Keithley 4200 semiconductor parameter analyzer, ranging from $240 \mathrm{~K}$ to $350 \mathrm{~K}$.

EL and PL measurements: For EL measurements, the bias was applied on the devices using a Keithley 2400 source meter. For PL measurements, the light source was a blue laser (405 nm). The spectra were detected using a light guide positioned close to the sample. The detector was a Newton EM-CCD Si array detector at $-60{ }^{\circ} \mathrm{C}$ with a Shamrock sr 303 i spectrograph from Andor Tech. 


\section{WILEY-VCH}

Wide Angle X-ray Scattering: Measurements were performed at the Stanford Synchrotron Radiation Lightsource (SSRL) on beamline 11-3. The incidence angle (0.12 degrees) was chosen to be above the critical angle of the film and data was recorded on a Mar 2D image plate detector. All measurements were performed in nitrogen with an X-ray energy of 12.7 $\mathrm{keV}$.

Small Angle X-ray Scattering: Measurements were performed on Beamline 7.3.3 at the Advanced Light Source at the Lawrence Berkeley National Laboratory. ${ }^{[46]}$ The experimental set-up was run in grazing incidence geometry and had a working distance of $3.82 \mathrm{~m}$. An Xray beam impinged onto the sample at a grazing angle above the critical angle of the polymer film, using incidence angles (0.12-0.18 degrees) but below the critical angle of the silicon substrate (0.22 degrees). The wavelength of X-rays used was $1.240 \AA(10 \mathrm{keV})$, and the scattered intensity was detected by using two-dimensional charge-coupled device (CCD) camera with image sizes of $2304 \times 2304$ pixels (Pilatus $6 \mathrm{M}$ detector). Background data was taken on a blank substrate (silicon substrate with $200 \mathrm{~nm}$ oxide) under the same conditions and exposure times of the polymer films. Data was processed using Nika 2D data reduction software. $^{[47]}$

\section{Supporting Information}

Supporting Information is available from the Wiley Online Library or from the author.

\section{Acknowledgements}

We are grateful to the Swedish Research Council (VR), the European Commission Marie Skłodowska-Curie actions, the Swedish Energy Agency, and the Knut and Alice Wallenberg foundation (KAW) for financial support.

Received: ((will be filled in by the editorial staff))

Revised: ((will be filled in by the editorial staff)) Published online: ((will be filled in by the editorial staff))

[1] J. Peet, J. Y. Kim, N. E. Coates, W. L. Ma, D. Moses, A. J. Heeger, G. C. Bazan, Nat. Mater. 2007, 6, 497. 


\section{WILEY-VCH}

[2] H.-Y. Chen, J. Hou, S. Zhang, Y. Liang, G. Yang, Y. Yang, L. Yu, Y. Wu, G. Li, Nat. Photonics 2009, 3, 649.

[3] Z. He, C. Zhong, S. Su, M. Xu, H. Wu, Y. Cao, Nat. Photonics 2012, 6, 591.

[4] F. Zhang, K. G. Jespersen, C. Björström, M. Svensson, M. R. Andersson, V. Sundström, K. Magnusson, E. Moons, A. Yartsev, O. Inganäs, Adv. Funct. Mater. 2006, 16, 667.

[5] A. J. Moulé, K. Meerholz, Adv. Mater. 2008, 20, 240.

[6] J. K. Lee, W. L. Ma, C. J. Brabec, J. Yuen, J. S. Moon, J. Y. Kim, K. Lee, G. C. Bazan, A. J. Heeger, J. Am. Chem. Soc. 2008, 130, 3619.

[7] K. Schmidt, C. J. Tassone, J. R. Niskala, A. T. Yiu, O. P. Lee, T. M. Weiss, C. Wang, J. M. J. Fréchet, P. M. Beaujuge, M. F. Toney, Adv. Mater. 2014, 26, 300.

[8] W. Chen, T. Xu, F. He, W. Wang, C. Wang, J. Strzalka, Y. Liu, J. Wen, D. J. Miller, J. Chen, K. Hong, L. Yu, S. B. Darling, Nano Lett. 2011, 11, 3707.

[9] S. J. Lou, J. M. Szarko, T. Xu, L. Yu, T. J. Marks, L. X. Chen, J. Am. Chem. Soc. 2011, 133, 20661.

[10] B. A. Collins, Z. Li, J. R. Tumbleston, E. Gann, C. R. McNeill, H. Ade, Adv. Energy Mater. 2013, 3, 65.

[11] J. T. Rogers, K. Schmidt, M. F. Toney, E. J. Kramer, G. C. Bazan, Adv. Mater. 2011, 23, 2284.

[12] X. Guo, M. Zhang, W. Ma, L. Ye, S. Zhang, S. Liu, H. Ade, F. Huang, J. Hou, Adv. Mater. 2014, 26, 4043.

[13] Y. Huang, E. J. Kramer, A. J. Heeger, G. C. Bazan, Chem. Rev. 2014, 114, 7006.

[14] L. Lu, L. Yu, Adv. Mater. 2014, 26, 4413.

[15] J. C. Blakesley, D. Neher, Phys. Rev. B 2011, 84, 075210.

[16] A. Rao, P. C. Y. Chow, S. Gélinas, C. W. Schlenker, C.-Z. Li, H.-L. Yip, A. K.-Y. Jen, D. S. Ginger, R. H. Friend, Nature 2013, 500, 435.

[17] E. R. Bittner, V. Lankevich, S. Gélinas, A. Rao, D. A. Ginger, R. H. Friend, Phys. Chem. Chem. Phys. 2014, 16, 20321.

[18] L. Ye, S. Zhang, L. Huo, M. Zhang, J. Hou, Acc. Chem. Res. 2014, 47, 1595.

[19] C. Tanase, P. W. M. Blom, D. M. de Leeuw, Phys. Rev. B 2004, 70, 193202.

[20] C. Tanase, E. J. Meijer, P. W. M. Blom, D. M. de Leeuw, Phys. Rev. Lett. 2003, 91, 216601.

[21] W. F. Pasveer, J. Cottaar, C. Tanase, R. Coehoorn, P. A. Bobbert, P. W. M. Blom, D. M. de Leeuw, M. A. J. Michels, Phys. Rev. Lett. 2005, 94, 206601.

[22] J. C. Blakesley, H. S. Clubb, N. C. Greenham, Phys. Rev. B 2010, 81, 045210.

[23] F. Gao, J. Wang, J. C. Blakesley, I. Hwang, Z. Li, N. C. Greenham, Adv. Energy Mater. 2012, 2, 956.

[24] J. C. Blakesley, F. A. Castro, W. Kylberg, G. F. A. Dibb, C. Arantes, R. Valaski, M. Cremona, J. S. Kim, J.-S. Kim, Org. Electron. 2014, 15, 1263.

[25] T. Kirchartz, Beilstein J. Nanotechnol. 2013, 4, 180.

[26] P. Stallinga, Adv. Mater. 2011, 23, 3356.

[27] M. P. Eng, P. R. F. Barnes, J. R. Durrant, J. Phys. Chem. Lett. 2010, 1, 3096.

[28] T. Kirchartz, B. E. Pieters, J. Kirkpatrick, U. Rau, J. Nelson, Phys. Rev. B 2011, 83, 115209.

[29] S. Foster, F. Deledalle, A. Mitani, T. Kimura, K.-B. Kim, T. Okachi, T. Kirchartz, J. Oguma, K. Miyake, J. R. Durrant, S. Doi, J. Nelson, Adv. Energy Mater. 2014, 4, 1400311.

[30] S. V. Novikov, D. H. Dunlap, V. M. Kenkre, P. E. Parris, A. V. Vannikov, Phys. Rev. Lett. 1998, 81, 4472.

[31] L. Mattias Andersson, W. Osikowicz, F. L. E. Jakobsson, M. Berggren, L. Lindgren, M. R. Andersson, O. Inganäs, Org. Electron. 2008, 9, 569. 


\section{WILEY-VCH}

[32] R. Coehoorn, W. F. Pasveer, P. A. Bobbert, M. A. J. Michels, Phys. Rev. B 2005, 72, 155206.

[33] L. H. Jimison, S. Himmelberger, D. T. Duong, J. Rivnay, M. F. Toney, A. Salleo, J. Polym. Sci. Part B Polym. Phys. 2013, 51, 611.

[34] S. Cho, J. K. Lee, J. S. Moon, J. Yuen, K. Lee, A. J. Heeger, Org. Electron. 2008, 9 , 1107.

[35] K. Tvingstedt, K. Vandewal, F. Zhang, O. Inganäs, J Phys Chem C 2010, 114, 21824.

[36] W. Gong, M. A. Faist, N. J. Ekins-Daukes, Z. Xu, D. D. C. Bradley, J. Nelson, T. Kirchartz, Phys. Rev. B 2012, 86, 024201.

[37] R. Shivanna, S. Shoaee, S. Dimitrov, S. K. Kandappa, S. Rajaram, J. R. Durrant, K. S. Narayan, Energy Environ. Sci. 2013, 7, 435.

[38] K. Tvingstedt, K. Vandewal, A. Gadisa, F. Zhang, J. Manca, O. Inganäs, J. Am. Chem. Soc. 2009, 131, 11819.

[39] K. Vandewal, S. Albrecht, E. T. Hoke, K. R. Graham, J. Widmer, J. D. Douglas, M. Schubert, W. R. Mateker, J. T. Bloking, G. F. Burkhard, A. Sellinger, J. M. J. Fréchet, A. Amassian, M. K. Riede, M. D. McGehee, D. Neher, A. Salleo, Nat. Mater. 2014, 13, 63.

[40] A. Melianas, V. Pranculis, A. Devižis, V. Gulbinas, O. Inganäs, M. Kemerink, Adv. Funct. Mater. 2014, 24, 4507.

[41] O. Glatter, O. Kratky, Small Angle X-Ray Scattering, Academic Press, 1982.

[42] A. Guinier, G. Fournet, Small Angle Scattering of X-Rays, John Wiley And Sons, New York, 1955.

[43] R. Noriega, J. Rivnay, K. Vandewal, F. P. V. Koch, N. Stingelin, P. Smith, M. F. Toney, A. Salleo, Nat. Mater. 2013, 12, 1038.

[44] F. Liu, W. Zhao, J. R. Tumbleston, C. Wang, Y. Gu, D. Wang, A. L. Briseno, H. Ade, T. P. Russell, Adv. Energy Mater. 2014, 4, 1301377.

[45] M. T. Dang, J. D. Wuest, Chem. Soc. Rev. 2013, 42, 9105.

[46] A. Hexemer, W. Bras, J. Glossinger, E. Schaible, E. Gann, R. Kirian, A. MacDowell, M. Church, B. Rude, H. Padmore, J. Phys. Conf. Ser. 2010, 247, 012007.

[47] J. Ilavsky, J. Appl. Crystallogr. 2012, 45, 324. 


\section{WILEY-VCH}
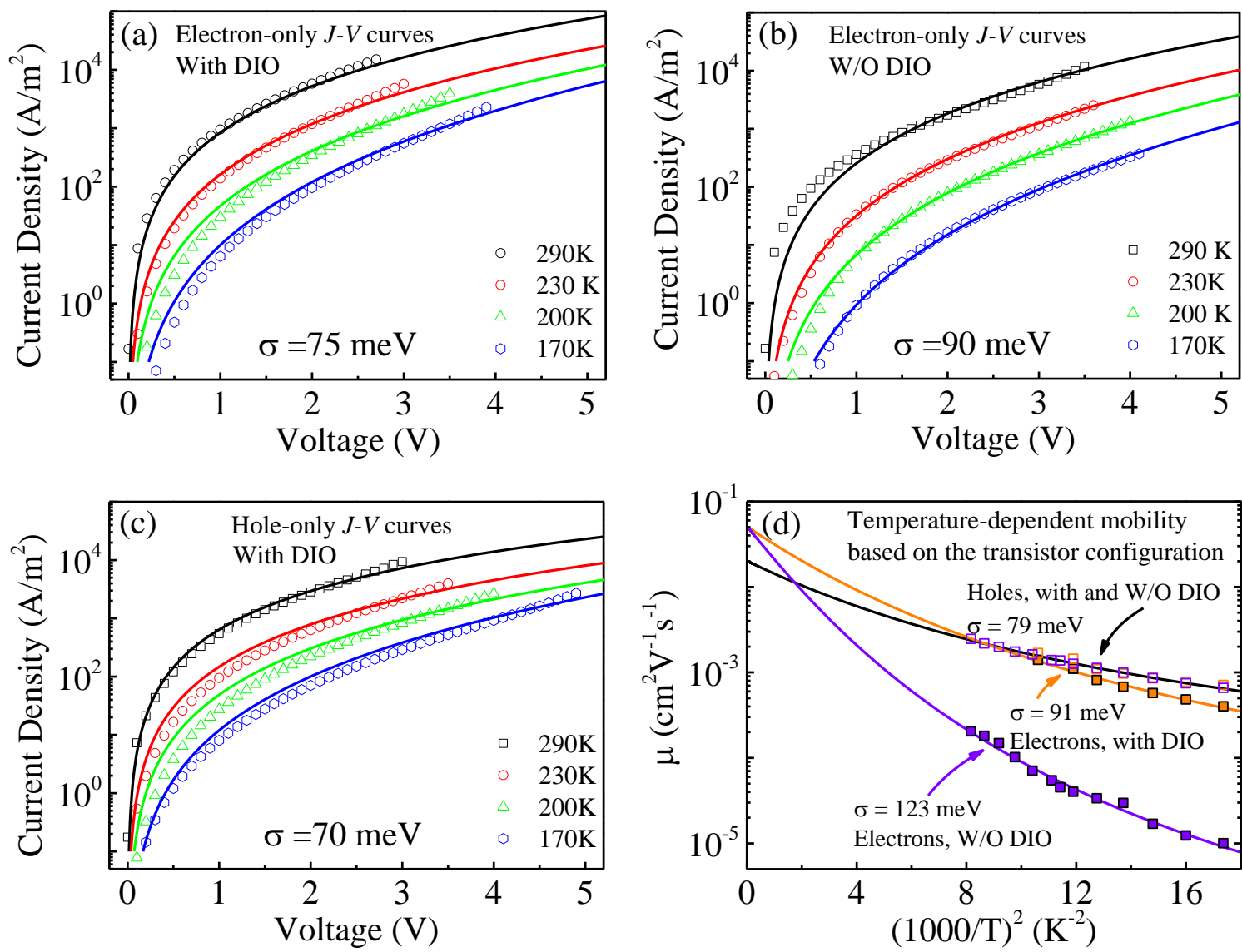

Figure 1. $J$ - $V$ curves of electron-only devices with (a) and without (b) DIO, where the symbols are the raw data and the solid lines are fitting curves. Disorder energy (for electrons) increases from 75 $\mathrm{meV}$ in the blend with DIO to $90 \mathrm{meV}$ in that without DIO. (c) Fitting curves (solid lines) for the holeonly curves of the device with DIO, giving a disorder energy of $70 \mathrm{meV}$. (d) Temperature-dependent mobility values based on the transistor configurations, showing that DIO decreases the energetic disorder for electrons without affecting the energetic ordering for holes, consistent with the findings based on the diode configuration. 


\section{WILEY-VCH}
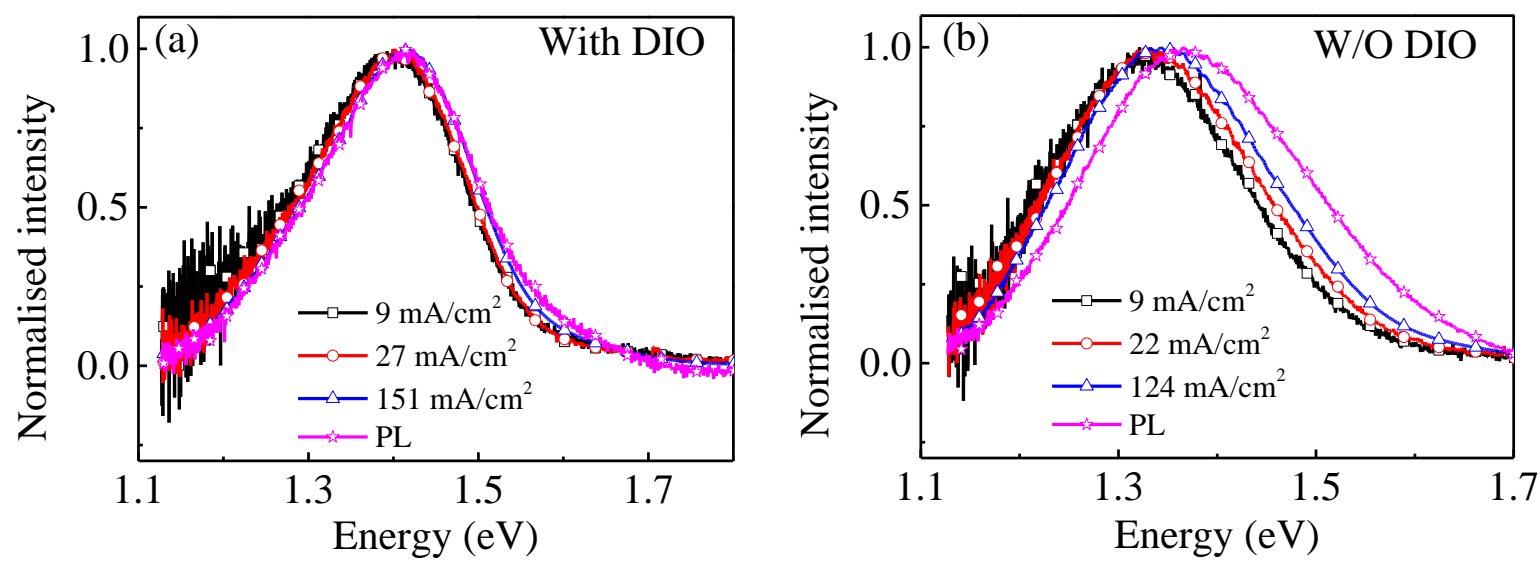

Figure 2. CT EL (at different bias) and PL spectra for the blends with (a) and without (b) DIO. The EL spectra in the blend with DIO show weaker dependence on the injected current. In addition, in the blend with DIO, the PL shows less blue shift compared with the EL. Both observations indicate smaller energetic disorder in the blend with DIO. 


\section{WILEY-VCH}
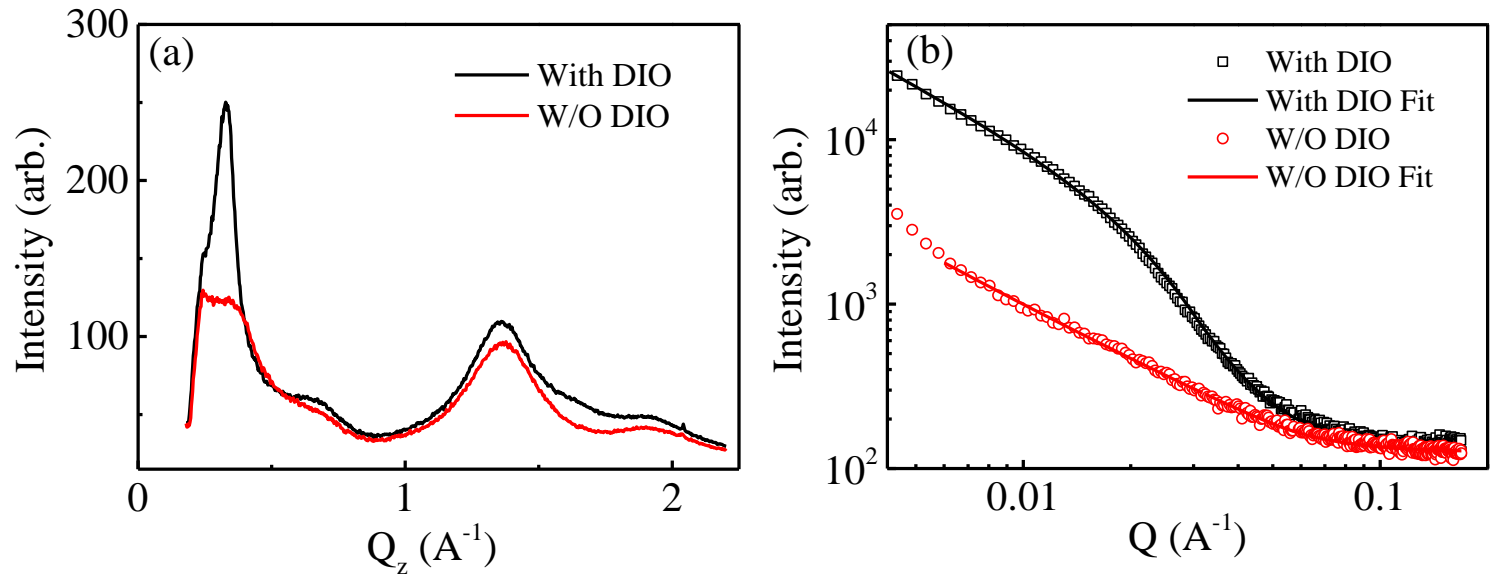

Figure 3. (a) WAXS line cuts for blends with and without DIO. Upon DIO addition the alkyl stacking peak intensity increases significantly and a small shoulder arising from the pi-stacking peak becomes visible near q 1.6 $\AA^{-1}$. (b) SAXS line cuts for the blends with and without DIO. The shoulder moves to lower q upon addition of DIO, indicating a larger average domain size. Fits using the Guinier-Porod model are shown as solid lines. 